\title{
Effect of fetal microchimeric cells on the development of postnatal depression
}

\author{
Demirhan $\mathrm{O}^{1 *}$, Ozturk N², Aydin N², Yildizdas HY', Demirbek B ${ }^{4}$, Uslu IN ${ }^{1}$, Gozet $\mathrm{Y}^{1}$ and Cakmak C ${ }^{2}$ \\ ${ }^{1}$ Department of Medical Biology and Genetics, Faculty of Medicine, Cukurova University, Adana, Turkey \\ ${ }^{2}$ University, Humanities and Social Sciences Faculty, Department of Psychology, Istanbul, Turkey \\ ${ }^{3}$ Department of Neonatology, Faculty of Medicine, Cukurova University, Adana, Turkey \\ ${ }^{4}$ Adana City Training and Research Hospital, Adana, Turkey
}

\begin{abstract}
Aim: Women are at the highest risk of depression during their childbearing years, and the birth of a child may precipitate a depressive episode in women. Fetal microchimerism (FMc), derived from fetal cells that persist after pregnancy, is usually evaluated by tests for male microchimerism (MMc) in women who gave birth to sons. We investigated FMc in women with sons and examined correlation with postnatal depression (PND) history, and this is one of the first studies in this area.

Methods: In this study, we performed real-time quantitative PCR to detect and quantify male DNA in whole peripheral blood of 64 women ( 32 healthy and 32 with PND), targeting the Y-chromosome-specific DYS14 gene sequence as a marker for microchimerism (Mc) of fetal origin.

Results: To verify the role of FMcCs in PND, 32 PND patients and 32 controlswere studied. A total of 32 women were studied of which 3 (9.4\%) tested positive for MMc, had given birth to at least one son. None of the 32 control healthy women were positive. FMc was found significantly more often in women with PND than in healthy women $(9.4 \%$ versus $0 \%$, respectively) ( $\mathrm{p}=0.005)$. About $41 \%$ of the risk for depression of PND women was familial, and smoking and drug usage habits was very high $(37.5 \%$ and $71.9 \%$, respectively) $(\mathrm{p}=0.005)$

Conclusion: MMc levels were higher in women with PND than in healthy women. Although the biological mechanisms are not precisely known, MMc presence in peripheral blood of women can be associated with PND. Genetic factors can also be one of the possible causes of PPD. Continued research on PND is needed to broaden the treatment alternatives in affected women.
\end{abstract}

\section{Introduction}

PND is defined as a nonpsychotic depressive illness of mild-tomoderate severity occurring in a mother during the first postnatal year, with most cases occurring in the first six months after delivery. The prevalence of NPD is generally reported as between $10 \%$ and $15 \%[1,2]$. All women are potential candidates for emotional upheaval during the postpartum period and women who are single, primiparous, or anxious or depressed during pregnancy, as well as those who have a history of mental illness or previous postpartum psychiatric disorders, have problems during labour or delivery, or have concurrent personal or social stresses, may be at increased risk. Since the effects of PND are known to go beyond the mother in that it also affects the partner and the child. Too often women disappear into the home postnatally and have limited outside contact for the crucial first months. Severe forms of depression and puerperal psychosis can lead some women to commit suicide to escape their emotions [3]. Low socioeconomic status; partner's habits, such as alcoholism; lack of education; marital conflict; unsupportive partner and psychological factors, such as antenatal depression, stressful life events or intimate partnerrecent and life events violences are all associated with PPD [4]. Depression has been observed to be higher in low-income and middle-income countries, ranging from $4.9 \%$ to $50.8 \%$ among mothers at $4-8$ weeks post partum [5]. The prevalence of depression among Asian women is $23.7 \%, 16.5 \%$ and $17.4 \%$ at 6 weeks, 3 months and 6 months after childbirth, respectively [6]. This evidence accumulates a body of knowledge that indicates PND is a global public health problem. Such research has been instrumental in demonstrating that the burden of morbidity is actually higher for low- and middle-income countries.

Whereas efforts to investigate psychosocial and epidemiological back ground of PPD have been extensive, the biological mechanisms and the genetic risk factors underlying PPD essentially remain unknown. However, research into the biological mechanisms of PPD is a challenging task. However, research into the biological mechanisms of PPD is a challenging task. We are putting forward a new hypothesis that will facilitate this challenge, which is not among the above mentioned possible causes of the disease. This is Mc. Regarding the characteristics and results of natural Mc, medical science needs more knowledge. Mc is defined by the presence within an individual of a low level of cells or DNA derived from a genetically different individual. Pregnancy is the most common and natural cause of chimerism, and bi-directional trafficking of hematopoietic cells occurs through the placenta. It is now known that during pregnancy, fetal-maternal microchimeric cells (F-MMcCs) can pass through tissues and organs such as peripheral blood, bone marrow, thymus, liver, lung, spleen, kidney, skin and brain.

${ }^{\star}$ Correspondence to: Osman Demirhan, Department of Medical Biology and Genetics, Faculty of Medicine, Cukurova University, 01330 Balcali, Adana, Turkey, E-mail: osdemir@cu.edu.tr

Received: March 01, 2019; Accepted: March 18, 2019; Published: March 21, 2019 
This manuscript discusses the applicability of Mc research in the field of psychiatric studies. The research into Mc, and more specifically F-MMc, is still in its infancy. In the literature there is no data regarding a connection between psychiatric illnesses and Mc. Male DNA, of presumed fetal origin, can be detected in the maternal circulation decades after delivery. FMc in maternal blood and tissues is typically identified using polymerase chain reaction (PCR) to identify Y-chromosome DNA sequences in the mother. Mc does not require the fetus to be male, but it is easier to distinguish the $\mathrm{Y}$ chromosome as a biomarker. The aim of the present study was to examine the presence of MMc in PND women using DNA extracted from cells from whole blood and report the association with potential sources of male cells.

\section{Patients, materials, and methods}

Study subjects: This work was supported by Cukurova University, Scientific Research Unit (Number 5369; Protocol 1707), and was approved by the University of Cukurova Ethic Board. All patients satisfied by Adana City Training and Research Hospital, Department of Psychiatry and Bakırkoy Mental and Neurological Hospital, and depressive symptoms were assessed with the Edinburgh Postnatal Depression Scale (EPDS). The American College of Rheumatology criteria for PND. Atotal of 64 women were studied, including 32 healthy controls and 32 patients with PND, who gave birth to a boy at their last birth, and had given birth to at least one son. Healthy women had no clinical or pathologic evidence of psychiatric diseases.

DNA isolation: To prepare samples for the quantitative PCR assay, DNA was isolated from patient's blood samples that by means of a High Pure PCR Template Preparation Kit (Rosche Life Science)

Real-time quantitative PCR: Real-Time reactions were carried out by LightCycler 480 Probes Master mix (Rosche Life Science). Reactions were performed by Rosche LightCycler 480 Real-Time PCR system. Primers used in Real-Time PCR reactions were designed by PrimerBLAST. DYS14 was used as reference gene. The DYS14 sequence (GenBank sequence accession number, X06325) was selected as the
Y-chromosome-specific sequence for use in the quantitative PCR (QPCR) assay. The reason for this choice was that, while single-copy genes, such as the SRY, can be reliably used in developing a QPCR assay. The DYS14, on the other hand, is believed to be strictly Y specific. Primers were designed and used with the following sequences: DYS14 forward primer, 5'-CATCCAGAGCGTCCCTGG-3'; and DYS14 reverse primer, 5'-TTCCCCTTTGTTCCCCAAA-3'. DYS14-probe sequence was 5'-6-FAM-TAAGTAACTGATGGGCAGCTCGGC(TAMRA)-3'. PCR cycling condition as follow: for denaturation, $10 \mathrm{~min} .95^{\circ} \mathrm{C}$. For amplication stage $(40 \mathrm{cycle}) ; 10 \mathrm{sec} .95^{\circ} \mathrm{C}, 1 \mathrm{~min} .56^{\circ} \mathrm{C}, 1 \mathrm{sec} .72^{\circ} \mathrm{C}$. For melting curve stage; $45 \mathrm{sec} .45^{\circ} \mathrm{C}$. For cooling stage $30 \mathrm{sec} .40^{\circ} \mathrm{C}$.

\section{Results}

Study subjects included 32 healthy women and 32 women with PND. A total of 32 PND women were tested for quantitative assessment of male DNA. Overall 3 out of 32 PND women (9.4\%) positive and 29 (90.6\%) of tested negative (Table 1, Figure 1).The all of healthy women who had given birth to a son had no detectable male DNA in the blood (Table 2). There was significant difference between women with PND and healthy women. MMc prevalence was significantly greater in women with PND than control groups (9.4\%, 0\%, respectively). Three healthy women with positive results had given birth to a son. These women 2 had given birth to a son and one women had 2 son. Fourteen of 32 (43.8\%) women with PND had similar psychological disturbances in previous pregnancies, and $18(56.3 \%)$ of them were found to have psychological discomfort in their family history. Twentythree (71.9\%) and $12(37.5 \%)$ of PND women were using drugs and smoking, respectively. In the control group, 6 of $32(18.8 \%)$ healthy women had psychological discomfort in their family history, and $3(9.4 \%)$ and 3 (9.4\%) women were using drugs and smoking, respectively.

Statistical analysis: Significant differences between the two groups were detected using the Chi square test for qualitative variables (Fisher exact test) and an unpaired t-test for continuous variables with a normal distribution or if not, a non-parametric test and a MannWhitney test. For all tests, statistical significance was defined as p,0.05.

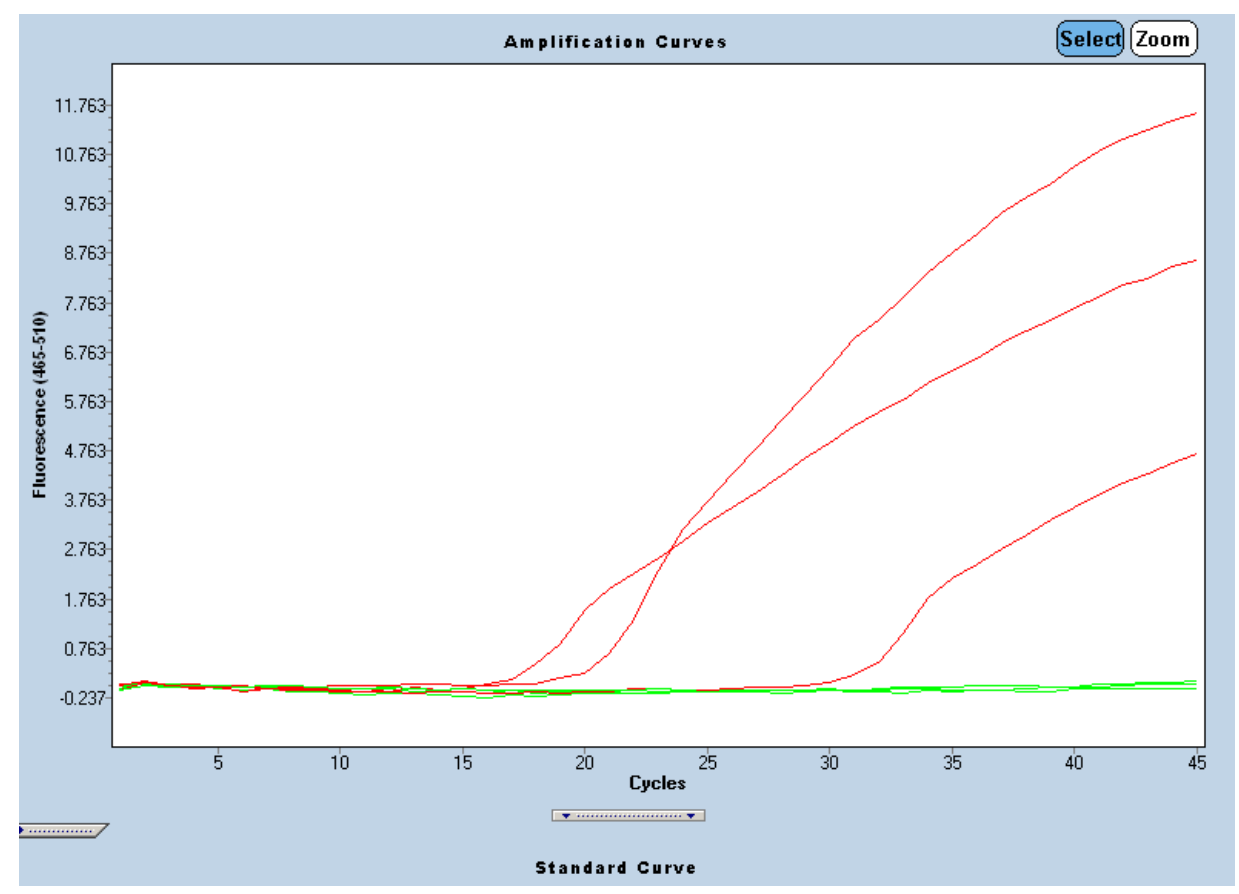

Figure 1. Positive amplification curves belonging to three patients 
Demirhan O (2019) Effect of fetal microchimeric cells on the development of postnatal depression

Table 1. Shows the distribution of male microchimerism according to reproductive, lifestyle and other variables in the women with PND

\begin{tabular}{|c|c|c|c|c|c|c|c|c|c|}
\hline Patient no & Age & $\begin{array}{c}\text { Total number of } \\
\text { children }\end{array}$ & $\begin{array}{c}\text { Number of male } \\
\text { children }\end{array}$ & $\begin{array}{c}\text { Gender of last } \\
\text { birth }\end{array}$ & Last birth / year & Drug use & $\begin{array}{l}\text { Similar disease } \\
\text { story in family }\end{array}$ & Smoking & FMcCs \\
\hline 1 & 36 & 1 & 1 & $\mathrm{M}$ & 2016 & + & - & - & - \\
\hline 2 & 30 & 3 & 2 & M & 2015 & - & + & + & - \\
\hline 3 & 29 & 1 & 1 & M & 2017 & + & + & + & - \\
\hline 4 & 33 & 1 & 1 & M & 2017 & - & - & + & - \\
\hline 5 & 27 & 1 & 1 & M & 2017 & - & - & - & + \\
\hline 6 & 38 & 1 & 1 & M & 2017 & - & + & - & - \\
\hline 7 & 33 & 1 & 1 & M & 2017 & + & - & - & - \\
\hline 8 & 35 & 1 & 1 & M & 2017 & + & - & + & - \\
\hline 9 & 37 & 1 & 1 & M & 2017 & - & - & + & + \\
\hline 10 & 29 & 5 & 2 & M & 2015 & + & - & - & + \\
\hline 11 & 37 & 2 & 2 & M & 2017 & + & - & - & - \\
\hline 12 & 42 & 3 & 1 & M & 2017 & + & - & + & - \\
\hline 13 & 39 & 1 & 1 & M & 2016 & + & + & - & - \\
\hline 14 & 27 & 1 & 1 & $\mathrm{M}$ & 2017 & + & - & - & - \\
\hline 15 & 27 & 1 & 1 & $\mathrm{M}$ & 2017 & - & - & - & - \\
\hline 16 & 40 & 4 & 4 & M & 2017 & - & + & - & - \\
\hline 17 & 24 & 1 & 1 & $\mathrm{M}$ & 2017 & + & - & - & - \\
\hline 18 & 37 & 2 & 2 & $\mathrm{M}$ & 2017 & + & - & + & - \\
\hline 19 & 37 & 2 & 1 & M & 2017 & + & + & + & - \\
\hline 20 & 38 & 1 & 1 & $\mathrm{M}$ & 2016 & + & - & + & - \\
\hline 21 & 34 & 1 & 1 & $\mathrm{M}$ & 2017 & + & - & - & - \\
\hline 22 & 24 & 2 & 2 & M & 2017 & - & + & - & - \\
\hline 23 & 21 & 3 & 2 & M & 2017 & + & + & + & - \\
\hline 24 & 37 & 1 & 1 & $\mathrm{M}$ & 2017 & + & + & - & - \\
\hline 25 & 35 & 3 & 1 & $\mathrm{M}$ & 2017 & + & + & + & - \\
\hline 26 & 33 & 2 & 1 & $\mathrm{M}$ & 2016 & - & - & - & - \\
\hline 27 & 28 & 2 & 1 & $\mathrm{M}$ & 2016 & + & + & + & - \\
\hline 28 & 37 & 3 & 2 & M & 2017 & + & - & - & - \\
\hline 29 & 41 & 3 & 1 & M & 2018 & + & - & - & - \\
\hline 30 & 38 & 2 & 1 & $\mathrm{M}$ & 2016 & + & + & + & - \\
\hline 31 & 36 & 1 & 1 & $\mathrm{M}$ & 2016 & + & - & - & - \\
\hline 32 & 33 & 1 & 1 & M & 2017 & + & + & - & - \\
\hline
\end{tabular}

Table 2. Shows the distribution according to control women tested negative or positive for male microchimerism

\begin{tabular}{|c|c|c|c|c|c|c|c|c|c|}
\hline Control no. & Age & $\begin{array}{c}\text { Total number of } \\
\text { children }\end{array}$ & $\begin{array}{c}\text { Number of male } \\
\text { children }\end{array}$ & $\begin{array}{c}\text { Gender of last } \\
\text { birth }\end{array}$ & Last birth / year & Drug use & $\begin{array}{l}\text { Similar disease } \\
\text { story in family }\end{array}$ & Smoking & FMcCs \\
\hline 1 & 23 & 1 & 1 & $\mathrm{M}$ & 2017 & - & - & + & - \\
\hline 2 & 21 & 1 & 1 & M & 2017 & - & - & - & - \\
\hline 3 & 30 & 2 & 2 & M & 2018 & - & + & - & - \\
\hline 4 & 27 & 2 & 1 & M & 2018 & - & - & - & - \\
\hline 5 & 23 & 3 & 1 & $\mathrm{M}$ & 2018 & - & - & - & - \\
\hline 6 & 29 & 2 & 1 & M & 2018 & - & - & - & - \\
\hline 7 & 36 & 3 & 3 & $\mathrm{M}$ & 2018 & - & - & - & - \\
\hline 8 & 33 & 2 & 1 & M & 2018 & - & - & - & - \\
\hline 9 & 29 & 2 & 1 & M & 2018 & - & + & - & - \\
\hline 10 & 34 & 5 & 2 & M & 2018 & - & - & - & - \\
\hline 11 & 32 & 1 & 1 & $\mathrm{M}$ & 2017 & - & - & - & - \\
\hline 12 & 27 & 3 & 1 & M & 2017 & - & - & - & - \\
\hline 13 & 23 & 1 & 1 & $\mathrm{M}$ & 2018 & + & + & + & - \\
\hline 14 & 23 & 2 & 1 & $\mathrm{M}$ & 2018 & - & - & - & - \\
\hline 15 & 27 & 3 & 1 & $\mathrm{M}$ & 2017 & - & - & - & - \\
\hline 16 & 25 & 1 & 1 & M & 2018 & - & - & - & - \\
\hline 17 & 28 & 1 & 1 & $\mathrm{M}$ & 2018 & + & - & - & - \\
\hline 18 & 23 & 2 & 2 & $\mathrm{M}$ & 2018 & - & - & - & - \\
\hline 19 & 38 & 3 & 3 & $\mathrm{M}$ & 2018 & - & - & - & - \\
\hline 20 & 34 & 3 & 2 & $\mathrm{M}$ & 2017 & - & - & - & - \\
\hline 21 & 30 & 2 & 2 & $\mathrm{M}$ & 2018 & - & + & - & - \\
\hline 22 & 25 & 1 & 1 & $\mathrm{M}$ & 2018 & - & - & - & - \\
\hline 23 & 27 & 2 & 2 & $\mathrm{M}$ & 2018 & - & - & - & - \\
\hline
\end{tabular}




\begin{tabular}{|c|c|c|c|c|c|c|c|c|c|}
\hline 24 & 30 & 2 & 1 & M & 2017 & - & - & - & - \\
\hline 25 & 38 & 2 & 1 & $\mathrm{M}$ & 2017 & - & - & - & - \\
\hline 26 & 35 & 2 & 1 & $\mathrm{M}$ & 2017 & - & + & + & - \\
\hline 27 & 34 & 1 & 1 & M & 2017 & - & - & & - \\
\hline 28 & 33 & 5 & 3 & M & 2017 & - & - & - & - \\
\hline 29 & 36 & 2 & 1 & M & 2017 & - & - & - & - \\
\hline 30 & 24 & 2 & 1 & $\mathrm{M}$ & 2018 & - & - & - & - \\
\hline 31 & 29 & 3 & 1 & $\mathrm{M}$ & 2017 & + & + & - & - \\
\hline 32 & 36 & 1 & 1 & $\mathrm{M}$ & 2017 & - & - & - & - \\
\hline
\end{tabular}

\section{Discussion}

In comparison with the vast epidemiological literature on PND, relatively few studies have examined the biological aspects of the disorder. However, research into the biological mechanisms of PND is a challenging task, and there are significant controversies about the disorder, including whether it is a distinct disorder or part of major depressive disorder, whether childbirth acts as a specific trigger for the onset of depression. Whereas efforts to investigate psychosocial and epidemiological background of PND have been extensive, the other risk factors underlying PND essentially remain unknown. Despite the research on risk factors for PND, understanding of related underlying mechanisms has not substantially progressed. The timing of onset of PND is an area of intense investigation.

To our knowledge, we are the first group to study the association between Mc and PND. This is one of the first study to quantitate the rate of McCs using a quantitative PCR approach. Thus, MMc quantification was estimated according to the source of chimerism, pregnancy, and compared to results obtained from healthy women in this study. In the present study, approximately, $44 \%$ of women with PND had similar psychological disturbances in previous pregnancies and $56.3 \%$ of them were also found to have psychological discomfort in their family history. The $19 \%$ of healthy women had also psychological discomfort in their family history. These ratios are quite high when compared to the control group $(\mathrm{p}=0.005)$. Also, the frequencyof PDN $(19 \%)$ in the family history of healthy women in our study is similar to the frequency of PDN (17.5\%) in our country [7]. These findings suggest that depression in women after birth may be familial or genetic. On the other hand, $71.9 \%$ and $37.5 \%$ of the patients and control group were using drugs and smoking, respectively. These ratios are also quite high when compared to the control group ( $\mathrm{p}=0.05$ ). The risk factors for PND has been identified that includes social, demographic, obstetric, biological, hormonal, psychiatric, and genetic features, as well as characteristics of the newborn child. It has also been proposed that the environmental risk for depression may be moderated by genetic factors. Genetic factors have been implicated as one of the probable causes of PPD, as determined by familial, twin, and adoption studies. It is estimated that about $40 \%$ to $50 \%$ of the risk for depression is genetic [8]. However, the specific mechanisms of genetic causality are not well understood [9]. and the relative contribution of various combinations of genetic and environmental factors to PND is as yet undetermined.

FMcCs were observed in almost all normal tissues of women with sons [10]. Other studies before the present have reported MMc prevalences of $43 \%$ and $24 \%$, respectively, among healthy women [11]. In a study, it assumed Y sequences in blood samples to originate from male pregnancies, with $70 \%$ of women testing positive [12]. Our results suggested higher prevalence and concentration of $\mathrm{MMc}$ in the blood of women with PND than the blood of women without disease. Nevertheless, the male DNA ratio in women with PND was found to be relatively low (positive in $9.4 \%$ of subjects). But we think it might be higher. The presence of microchemical cells was less than we expected. We assumed MMc to originate from pregnancies with a male fetus. Contrary to what we expected, we found no obvious association between the number of live-born boys and detection of MMc. Possible explanations include more unrecognized pregnancies, the small amount of blood sample taken (DNA at micron level), the duration of the postpartum period, personal factors or the employment of a test with little improved sensitivity and/or reduced specificity. At the same time, differences in technique include whether an assay was qualitative or quantitative. Another important reason for the low male DNA level in our patients may be the placement of the fetal cells in the mother's brain. For this reason, fewer cells may have been caught in the mother's blood.

A similar absence of association between parity and FMc was also recently reported by Gammill et al. [13] However, they also showed significantly reduced odds of MMc with increasing parity. In our results, although the incidence of male DNA in women with PND is low. Even this rate shows that quantitative DNA was related to PND. Accumulating date from biology and medicine show that natural chimerism is astonishingly frequent and may affect human conditions. Presently, the temporality, proportion and the outcome of male microchimerism is rarely known.

FMc is a common occurrence across placental mammals, the effects of such cells on maternal health remain a topic of fierce debate in the biological community. Cells derived from the fetus which can persist in maternal tissues for decades after a child is born have been associated with both protection and increased susceptibility to a range of afflictions, including cancer and autoimmune diseases like rheumatoid arthritis. However, it has been suggested that there may be a relationship between postpartum disorders and Mc. The etiology of the majority of mental illnesses is unknown. McCs have been detected in the brain tissue of rats and fetuses and in other tissues and organs as well. That McCs can penetrate the human brain and reside in the brain was first indicated by murine studies that showed the presence of both foreign cells and DNA in mouse brains $[14,15]$. In a study indicate that fetal DNA and likely cells can cross the human blood-brain barrier and reside in the brain, and male microchimerism is frequent and widely distributed in the human female [16].

It is currently not possible to meaningfully compare Mc prevalence or concentration in human brain to other tissues. The most likely source of MMc in female brain is acquisition of FMc from pregnancy with a male fetus. At present, the biological significance of harboring $\mathrm{Mc}$ in the human brain requires further investigation. The literature has not progressed significantly beyond a speculative role for the longterm existence of fetal cells in women with autoimmune diseases. The main basis of this hypothesis Mc, which is known to play an etiological role in autoimmune diseases, may also be the causative factor in the development of schizophrenia and postpartum psychosis. There is no consensus on whether the McCs that migrate from mother to fetus is to 
repair some pathology in the body or is the cause of any possible future pathology. In the etiological explanations of psychotic disorders; it has been shown that there is no consensus on what some processes from the fetal daylight do not go well and what is the triggering factor [17]. It is likely that FCs play each of these roles at various times. But, according to this, it has been suggested that the development of PND and psychosis may be triggered by the effect of the central nervous system on the immune response to McCs that pass to the mother and possibly to the critical level [17]. Even though there have been many studies on $\mathrm{Mc}$ in medicine, no study have been performed on the field of psychiatry. Our findings are suggested that there may be a relationship between PND and Mc. We believe that Mc may be an important alternative explanation to the etiology of chronic degenerative psychiatric diseases and postpartum clinical conditions. In this context, we hypothesized that Mc, which plays an important role in systemic diseases such as systemic sclerosis from thyroid disorders, may play a role in the etiology of some psychiatric disorders.

In a study conducted in our country, the prevalence of PND was found to be $17.5 \%$ [8]. There was a significant correlation between high parity and PND. According to this, the prevalence of PND in children with pre-existing cases was found to be statistically significantly higher than those without [7]. The increased risk of impairment due to an increase in the number of births may be related to the increased risk of chimeric cell transplantation from the baby to the mother. according to this; the immune response to the fetal microchimeric triplet in the mother may have been triggered by the effect on the central nervous system, PND and the development of psychosis [17]. Stem cell studies have shown that somatic cells differentiate with various viruses and chemical agents and can transform into stem cells [18]. Fetal stem and progenitor cells in mice can be found in several different regions of the mother's brain and these cells undergo neural cell-like transformation [15]. At the same time, fetal stem and progenitor cells have been shown to integrate into several regions of the brain and differentiate into maternal neuronal cells, participate in axonal and dendritic formations, and participate in adult neurogenesis. In this study; fetal stem cell infusion may have the potential to trigger neurodegenerative diseases of the central nervous system. According to this result; it is possible that somatic cells of the mother should be organized so that they can form a part of the fetal brain tissue by dedifferentiation and repetition of differentiation. When it is thought that a very small number of cells can control a neurotransmitter system, such as locus serendipitous, it seems possible that some of the mental illnesses may occur by affecting the development of brain tissue of the microchemical cells passing through the mother.

There are various researches on the role of Mc in the etiology of autoimmune diseases. It is suspected that schizophrenia patients may also have an autoimmune cause. In schizophrenia patients, however, no specific antigen was found to cause disease and brain changes in autoantibody isolation studies of tissue components of the central nervous system. As schizophrenia can cause various central nervous system tissue damage, the release of brain antigens and the formation of brain autoantibodies to central nervous system tissues can be a consequence of the central nervous system pathology $[19,20]$. FMcCs located between the maternal brain cells may interfere with accurate neuronal transmission of accurate information between neuronal cells. Until the mother's reaction to the chimeric cells in the central nervous system has reached a certain critical level and the psychosis table has emerged, it has been shown that either mild neurological manifestations or psychodynamic prodromal signs (social withdrawal, reduced productivity, somatic and obsessive strokes, low scores from tests, etc.). Numerous biological pathways may act in concert to increase the risk of PND, and it is also reasonable to assume that different biological mechanisms may come into play in individual women.

\section{Conclusion}

The present study is the first to establish a link between MMc and among women with PND. These findings can be interpreted as a pioneer basic data supporting the hypothesis that we have proposed. We believe that Mc may be an important alternative explanation to the etiology of chronic degenerative psychiatric diseases and postpartum clinical conditions. The biological significance of harboring Mc in the PND women requires further investigation. Genetic factors can also be one of the possible causes of PPD. We think that Mc may be explanatory especially for psychotic disorders and mental illnesses developed during postpartum period and the work to be done in this direction is an important need.

\section{References}

1. Moses-Kolko EL, Roth EK (2004) Antepartum and postpartum depression: healthy mom, healthy baby. J Am Med Womens Assoc 59: 181-191. [Crossref]

2. O'Hara MW, Swain AM (1996) Rates and risk of postpartum depression-a metaanalysis. Int Rev Psychiatry 8: 37-54. [Crossref]

3. Almond P (2009) Postnatal depression: A global public health perspective. Royal Society for Public Health 129: 221-227.

4. American Psychiatric Association (2000) Diagnostic and statistical manual of mental disorders. 5th edn. Washington: DC, USA.

5. Norhayati MN, Hazlina NH, Asrenee AR (2015) Magnitude and risk factors for postpartum symptoms: a literature review. J Affect Disord 175: 34-52. [Crossref]

6. Roomruangwong C (2011) Perinatal depression in Asian women: prevalence, associated factors, and cultural aspects. Asian Biomedicine 5: 179-193.

7. Eren TI (2007) Postpartum depresyon: Prevalansi ve sosyodemografik risk faktorleri (Uzmanlık Tezi). İstanbul, Haydarpaşa Numune Egitim ve Arastırma Hastanesi.

8. Shapiro GD, Fraser WD, Seguin JR (2012) Emerging risk factors for postpartum depression: Serotonin transporter genotype and omega-3 fatty acid status. Canadian Journal of Psychiatry 57: 704-712. [Crossref]

9. Leung BM, Kaplan BJ (2009) Perinatal depression: prevalence, risks, and the nutrition link-a review of the literature. J Am Diet Assoc 109: 1566-1575. [Crossref]

10. Nemescu D, Onofriescu M (2008) The impact of fetal cells persistence in maternal organism. Gineco Ro 4: 212-216.

11. Gadi VK, Nelson JL (2007) Fetal microchimerism in women with breast cancer Cancer Res 67: 9035-9038. [Crossref]

12. Gammill HS, Guthrie KA, Aydelotte TM, Waldorf KM, Nelson JL, et al. (2010) Effect of parity on fetal and maternal microchimerism: interaction of grafts within a host? Blood 116: 2706-2712. [Crossref]

13. Kamper-Jorgensen M, Hjalgrim H, Andersen ANN, Gadi WK, Tjonneland A, et al. (2014) Male microchimerism and survival among women. International Journal of Epidemiology 43: 168-173.

14. Gammill HS, Guthrie KA, Aydelotte TM, Adams Waldorf KM, et al. (2010) Effect of parity on fetal and maternal microchimerism: interaction of grafts within a host? Blood 116: $2706-2712$

15. Kaplan J, Land S (2005) Influence of maternal-fetal histocompatibility and MHC zygosity on maternal microchimerism. J Immunol 174: 7123-7128. [Crossref]

16. Tan XW, Liao H, Sun L, Okabe M, Xiao ZC, et al. (2005) Fetal microchimerism in the maternal mouse brain: a novel population of fetal progenitor or stem cells able to cross the blood-brain barrier? Stem Cells 23: 1443-1452.

17. Chan WFN, cile Gurnot C, Montine TJ, Sonnen JA, Guthrie KA, et al. (2012) Male Microchimerism in the Human Female Brain. PLOS ONE 7: e45592. [Crossref]

18. Demirbek B (2010) Psikotik bozukluklarda mikrokimerizm etyolojik bir faktor olabilir mi? Bir hipotez onerisi. Psikiyatride Guncel Yaklasimlar 2: 417-420. 
Demirhan O (2019) Effect of fetal microchimeric cells on the development of postnatal depression

19. Cetinkaya DU (2009) Mezenkimal kok hucreler. In Kok Hucre Biyolojisi ve Klinik Uygulamalar (Ed TUBA Kok Hucre Calisma Grubu): 53-62. Ankara, TUBA.
20. Brown AS, Bresnahan M, Susser ES (2005) Schizophrenia: Environmental epidemiology In Kaplan \& Sadock's Comprehensive Textbook of Psychiatry, 8th Edition (Eds BJ Sadock, VA Sadock):1372-1381. Baltimore, Lippincott Williams\&Wilkins.

Copyright: (C2019 Demirhan O. This is an open-access article distributed under the terms of the Creative Commons Attribution License, which permits unrestricted use, distribution, and reproduction in any medium, provided the original author and source are credited. 